\title{
Novel electrocardiographic features in carriers of hypertrophic cardiomyopathy causing sarcomeric mutations
}

\author{
Jalanko, Mikko
}

2018

Jalanko , M , Heliö , T , Mustonen , P , Kokkonen , J , Huhtala , H , Laine , M , Jääskeläinen , P , Tarkiainen , M , Lauerma , K, Sipola , P , Laakso , M , Kuusisto , J \& Nikus , K 2018 , ' Novel electrocardiographic features in carriers of hypertrophic cardiomyopathy causing sarcomeric mutations ' , Journal of Electrocardiology , vol. 51 , no. 6 , pp. 983-989 . https://doi.org/10.1016/j.jelectroc

http://hdl.handle.net/10138/308867

https://doi.org/10.1016/j.jelectrocard.2018.07.009

publishedVersion

Downloaded from Helda, University of Helsinki institutional repository.

This is an electronic reprint of the original article.

This reprint may differ from the original in pagination and typographic detail.

Please cite the original version. 


\title{
Novel electrocardiographic features in carriers of hypertrophic cardiomyopathy causing sarcomeric mutations
}

\author{
Mikko Jalanko a,*, Tiina Helïö ${ }^{a}$, Pirjo Mustonen ${ }^{\mathrm{b}}$, Jorma Kokkonen ${ }^{\mathrm{b}}$, Heini Huhtala ${ }^{\mathrm{c}}$, Mika Laine ${ }^{\mathrm{a}}$, \\ Pertti Jääskeläinen ${ }^{\mathrm{d}}$, Mika Tarkiainen ${ }^{\mathrm{e}}$, Kirsi Lauerma ${ }^{\mathrm{f}}$, Petri Sipola ${ }^{\mathrm{e}}$, Markku Laakso ${ }^{\mathrm{g}}$, \\ Johanna Kuusisto ${ }^{\mathrm{g}}$, Kjell Nikus ${ }^{\mathrm{h}}$ \\ ${ }^{a}$ Heart and Lung Center, Department of Cardiology, Helsinki University Hospital and University of Helsinki, Helsinki, Finland \\ b Central Hospital of Central Finland, Jyväskylä, Finland \\ c Faculty of Social Sciences, University of Tampere, Finland \\ d Heart Center, Kuopio University Hospital, Kuopio, Finland \\ e Department of Radiology, Kuopio University Hospital, Kuopio, Finland \\ ${ }^{\mathrm{f}}$ Department of Radiology, Helsinki University Hospital, Helsinki, Finland \\ g Center for Medicine and Clinical Research, University of Eastern Finland and Department of Medicine, Kuopio University Hospital, Kuopio, Finland \\ ${ }^{\mathrm{h}}$ Heart Center, Department of Cardiology, Tampere University Hospital, and Faculty of Medicine and Life Sciences, University of Tampere, Finland
}

\section{A R T I C L E I N F O}

\section{Keywords:}

Hypertrophic cardiomyopathy

Mutation carrier

Electrocardiography

\begin{abstract}
A B S T R A C T
Objectives: The sensitivity and specificity of the conventional 12-lead ECG to identify carriers of hypertrophic cardiomyopathy (HCM) - causing mutations without left ventricular hypertrophy (LVH) has been limited. We assessed the ability of novel electrocardiographic parameters to improve the detection of HCM mutation carriers. Methods: We studied 140 carriers (G+) of the TPM1-Asp175Asn or MYBPC3-Gln1061X pathogenic variants for HCM: The $\mathrm{G}+/ \mathrm{LVH}+$ group $(n=98)$ consisted of mutation carriers with LVH and the G+/LVH - group $(n=$ 42 ) without LVH. The control group consisted of 30 subjects. The standard 12-lead ECG was comprehensively analyzed and two novel ECG variables were introduced: RV1 $<$ RV2 $>$ RV3 and septal remodeling. A subset of 65 individuals underwent cardiac magnetic resonance imaging and 2D strain echocardiography.

Results: Conventional major ECG criteria were sensitive (90\%) and specific (97\%) in identifying G+/LVH+ subjects. RV1 $<\mathrm{RV} 2>\mathrm{RV} 3$ and septal remodeling were more prevalent in the $\mathrm{G}+/ \mathrm{LVH}-$ subjects compared to the control group (33\% vs $3 \%, \mathrm{p}=0.005$ and $45 \%$ vs $3 \%, \mathrm{p}<0.001$, respectively). The combination of $\mathrm{RV} 1<\mathrm{RV} 2>\mathrm{RV} 3$ and $\mathrm{Q}$ waves and repolarization abnormalities (QR) differentiated between the $\mathrm{G}+/ \mathrm{LVH}$ - subjects and the control group with a sensitivity of $52 \%$ and specificity of $97 \%$. The combination of septal remodeling and QR differentiated between $\mathrm{G}+/ \mathrm{LVH}$ - subjects and the control group with a sensitivity of $64 \%$ and specificity of $97 \%$.

Conclusions: The novel ECG-parameters RV1 $<\mathrm{RV} 2>\mathrm{RV} 3$ and septal remodeling were effective in identifying $\mathrm{G}+/$ LVH - subjects and could be useful in the diagnostics of new suspected HCM patients and in the screening and follow-up of HCM families.
\end{abstract}

(c) 2018 Elsevier Inc. All rights reserved.

\section{Introduction}

Hypertrophic cardiomyopathy (HCM) is the most common inherited cardiac disease and one of the leading causes of sudden cardiac death in young people and competitive athletes [1]. To date, over 1500 pathological gene variants in eight genes encoding sarcomeric proteins have been found [2]. In probands with clinical HCM, however, a pathogenic variant is identified in approximately $30-60 \%$ of cases $[2,3]$. The diagnostic accuracy of identifying individuals at risk for

\footnotetext{
* Corresponding author at: Helsinki University Hospital, Heart and Lung Center, Department of Cardiology, Haartmaninkatu 4, PB 340, FIN-00029 HUS, Finland.

E-mail address: mikko.jalanko@hus.fi (M. Jalanko).
}

developing HCM in families of probands with no identified variant needs improvement. Disease development in carriers of pathogenic variants without $\mathrm{LVH}(\mathrm{G}+/ \mathrm{LVH}-)$ should also be monitored regularly [3]. The standard 12-lead electrocardiogram (ECG) is an inexpensive and universally available method used widely in the diagnostics of HCM. Common ECG findings in HCM include LVH, abnormal Q waves and repolarization abnormalities such as T-wave inversions, ST-segment depression, and prolonged QT [4-8]. However, the sensitivity and specificity of the ECG to identify G+/LVH - subjects have been limited and the prior published data are scarce [4,5,8-10]. In this study our objective was to investigate previously unreported ECG abnormalities of R-wave amplitude distribution and QRS configuration in leads V1-V3 to identify carriers of a pathogenic HCM variant with or without 
echocardiographic LVH. In addition, we aimed to correlate the ECG findings of mutation carriers with comprehensive imaging data in a subset of 65 subjects.

\section{Material and methods}

\section{Patients}

The patients were recruited from families with HCM evaluated at Kuopio and Helsinki University Hospitals and the Central Hospital of Central Finland. The study population consisted of 140 subjects carrying either one of two Finnish founder mutations causing HCM: The Gln1061X mutation in MYBPC3 or the Asp175Asn mutation in TPM1. Mutation carriers with documented maximum LV wall thickness $(\mathrm{MWT}) \geq 13 \mathrm{~mm}$ on echocardiography comprised the $\mathrm{G}+/ \mathrm{LVH}+$ group ( $n=98$ ). Mutation carriers without the HCM phenotype (MWT $<13 \mathrm{~mm}$ ) were classified as the $\mathrm{G}+/ \mathrm{LVH}-$ group $(n=42)$. The control group $(n=30)$ consisted of relatives of individuals with either one of the two variants, with a structurally normal heart by echocardiography and who were not carriers of the two studied variants. The Ethics Committees at the Kuopio and Helsinki University Hospitals approved the study protocol. The study conforms to the principles outlined in the Declaration of Helsinki.

\section{Laboratory methods}

Venous blood samples were collected and the plasma concentrations of the N-terminal portion of brain natriuretic peptide (NT-proBNP) were determined with immunoassays utilizing antisera directed to NTproBNP. The sensitivity of the assay was $40 \mathrm{pmol} / \mathrm{L}$. The genetic studies were performed in the Genome Center of Eastern Finland, as previously described [11].

\section{ECG analysis}

All standard 12-lead ECGs, recorded at $50 \mathrm{~mm} / \mathrm{s}$ speed and $10 \mathrm{~mm} / \mathrm{mV}$ gain, were analyzed manually by one investigator (KN) blinded to the clinical data. The amplitudes of the R and S waves were measured from all the 12 standard ECG leads. The ST segment was considered elevated or depressed if it was $0.5 \mathrm{~mm}$ or more above or below the baseline, respectively, determined by drawing a line between subsequent PR intervals. The T wave was considered negative if it was $1 \mathrm{~mm}$ or more below the baseline. We did not include patients with left bundle branch block or pacemaker ECG.

In addition to the amplitudes of the $\mathrm{R}$ and $\mathrm{S}$ waves in all the $12 \mathrm{ECG}$ leads, the following previously described ECG parameters were recorded: 1) pathological Q waves: any Q wave $\geq 40 \mathrm{~ms}$ in duration, or $\geq 3 \mathrm{~mm}$ deep, or $\mathrm{Q} / \mathrm{R}$-ratio $\geq 0.25$, in $\geq 2$ contiguous leads except lead aVR [12], 2) absence of normal $Q$ wave in V5-V6 [13], 3) highest $R$ wave in the precordial leads outside of leads V4-V6 [14], 4) LVH according to the Sokolow-Lyon criteria (SV1 + maximal R wave amplitude of V5 or V6 $\geq 35 \mathrm{~mm}$ ), 5) LVH according to the Cornell voltage-duration product defined as: QRS-duration $(\mathrm{ms}) \times(\operatorname{RaVL}[\mathrm{mm}]+\mathrm{SV} 3[\mathrm{~mm}])$. For women $+8 \mathrm{~mm}$ was added to the voltage. The product was positive for LVH with a cutoff of $\geq 2440$ [15], 6) QRS duration $>100 \mathrm{~ms}, 7$ ) RI+SIII $>25$ mm [16], 8) RI+SIII-RIII-SI $>17$ mm [17], 9) $\mathrm{S}>\mathrm{R}$ in V4 [18], 10) Romhilt-Estes point score $\geq 4$ suggesting LVH [19], 11) P-terminal force (PTF; negative portion of the P-wave in lead V1 $\geq 0.04 \mathrm{~mm} \cdot \mathrm{s}$ ), 12) STsegment depression $\geq 0.5 \mathrm{~mm}$ in $\geq 2$ contiguous leads, 13) T-wave inversion $\geq 1 \mathrm{~mm}$ in $\geq 2$ contiguous leads, except for leads aVR and V1, 14) frontal plane left, right or superior axis deviation, 15) fragmented QRS (as defined by Das and Zipes) [20], 16) poor R-wave progression (PRWP) [21], or 17) reverse R-wave progression in leads V1-V3 [21], 18) rhythm other than sinus, 19) prolonged PR interval, 20) prolonged QTc interval $\geq 440 \mathrm{~ms}$ (Bazett formula).
The electrocardiographic parameters proposed by McKenna et al. were considered as major criteria for HCM detection, including pathological Q waves, LVH by Romhilt-Estes score $\geq 4$, and T-wave inversion $(>3 \mathrm{~mm})$ in at least two leads [22]. Composite ECG criteria were analyzed to aid in clinical identification of $\mathrm{G}+/ \mathrm{LVH}+$ and LVH - subjects (Table 1): Q waves and repolarization abnormalities (QR; repolarization abnormalities $=\mathrm{T}$-wave inversion and/or ST-segment depression and/or prolonged QTc) and hypertrophy by Cornell voltage-duration product and/or Sokolow-Lyon criteria and/or Romhilt-Estes score $\geq 4$.

In addition, we studied two novel ECG parameters. First, $\mathrm{RV} 1<\mathrm{RV} 2>\mathrm{RV} 3$, defined as the $\mathrm{R}$ wave peak being higher in $\mathrm{V} 2$ compared to V1 and V3 (Fig. 1), which we decided to include based on experience from clinical practice and from pathophysiological processes involved in HCM. Second, a composite ECG criterion named septal remodeling was analyzed, which we have previously used in a study on dilated cardiomyopathy [23]. Septal remodeling was positive with at least one of the following findings in the leads V1-V3: (1) pathological Q waves in $\geq 2$ contiguous leads, (2) QRS fragmentation in $\geq 2$ leads, (3) PRWP accompanied by QRS fragmentation, or (4) disorderly distributed R-wave amplitudes, either $R V 2>R V 3$ or $R V 1>R V 2$. The possibility of lead switch was searched for by analyzing the morphology of the P and S waves in the precordial leads; no suspicious cases were observed.

\section{Echocardiography}

All subjects underwent conventional echocardiographic assessment with emphasis on changes due to HCM. Measurements were made according to ACC/AHA/ASE guidelines. MWT was measured from 2D images with optimal beam alignment to the interrogated wall.

Table 1

Baseline variables of the study groups.

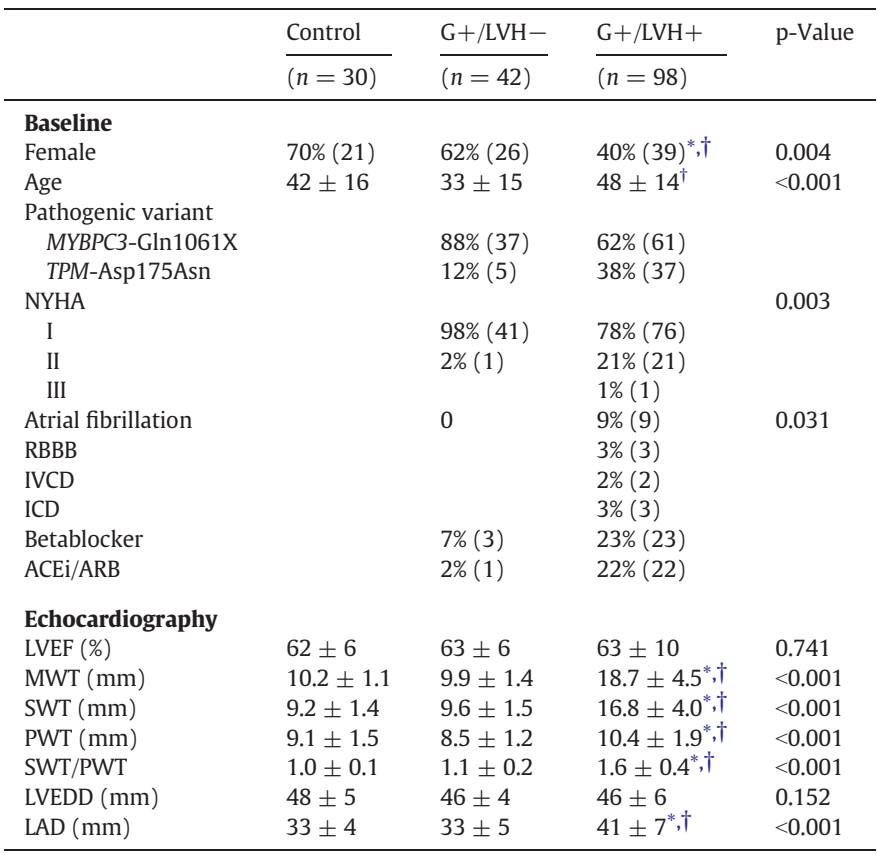

Presented as mean \pm SD or percentage and number of subjects in parentheses. $\mathrm{ACEi}=$ angiotensin converting enzyme inhibitor, $\mathrm{ARB}=$ angiotensin receptor blocker, $\mathrm{ICD}=$ implantable cardioverter defibrillator, IVCD = intraventricular conduction delay, LAD = left atrial dimension, LVEDD = left ventricular end-diastolic dimension, LVEF = left ventricular ejection fraction, MWT $=$ maximal wall thickness, SWT $=$ septal wall thickness, $\mathrm{PWT}=$ posterior wall thickness, RBBB $=$ right bundle branch block, SWT/ $\mathrm{PWT}=$ septal to posterior wall thickness ratio.

$* \mathrm{p}<0.05$ for difference between indicated group and control group.

$\dagger \mathrm{p}<0.05$ for difference between $\mathrm{G}+/ \mathrm{LVH}+$ and $\mathrm{G}+/ \mathrm{LVH}-$ groups. 
A

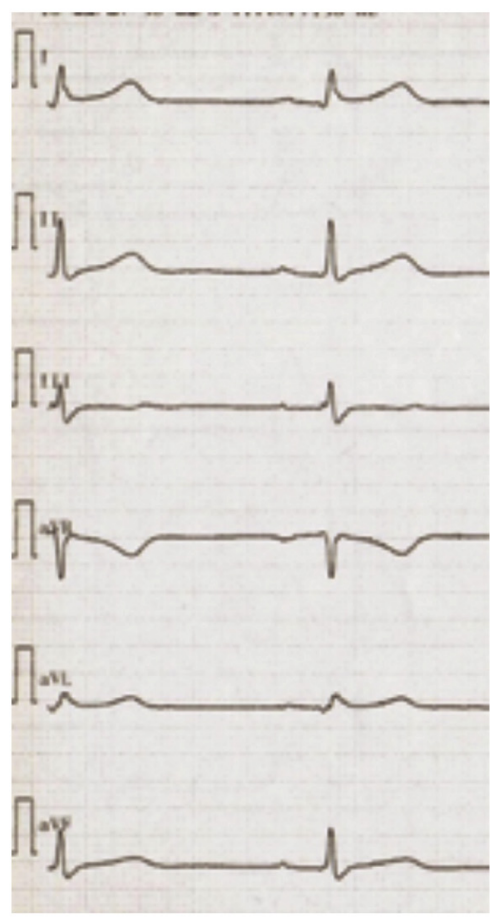

B

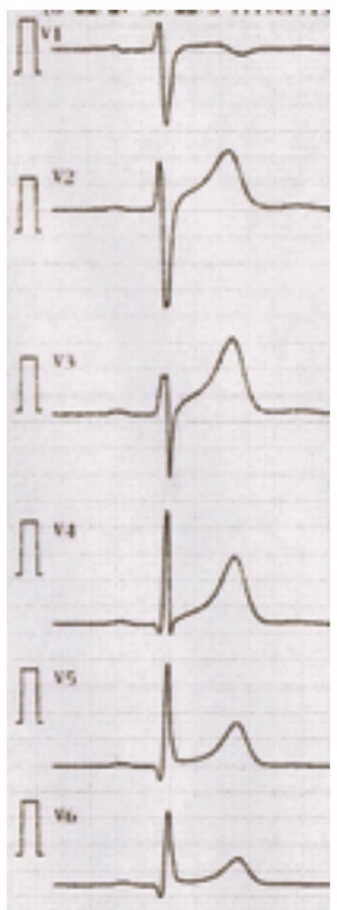

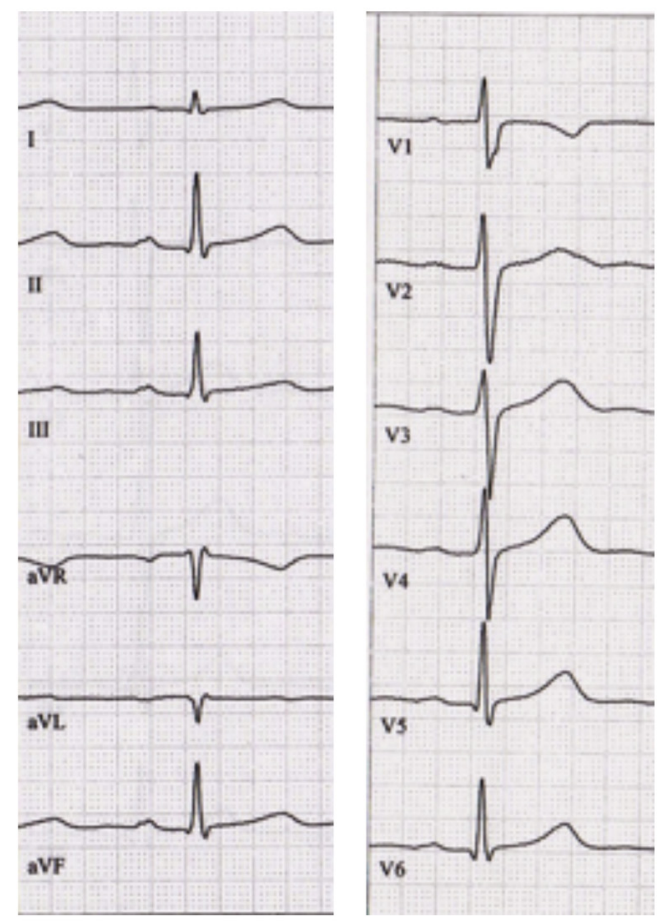

C

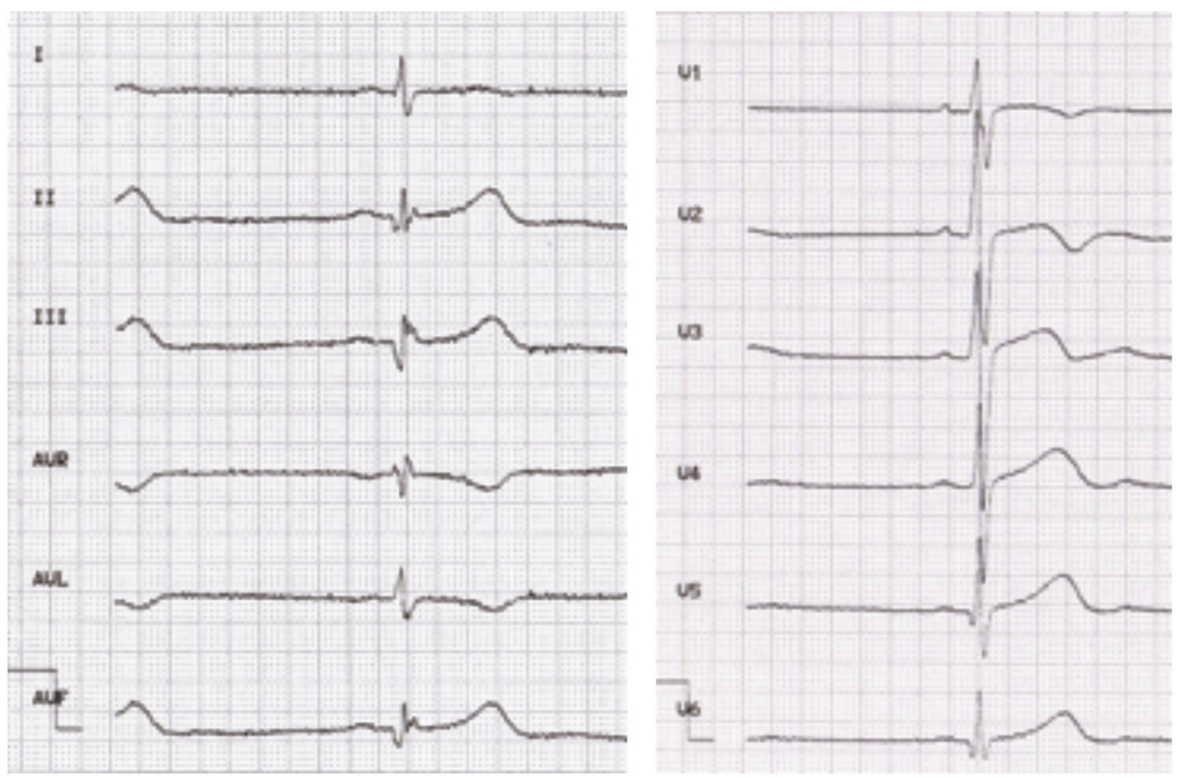

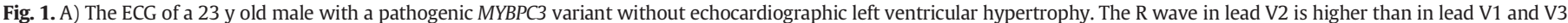

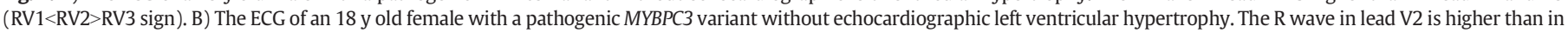

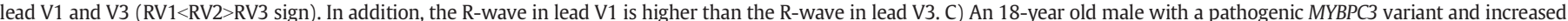

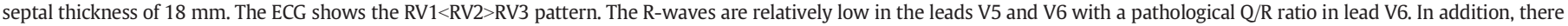
are Q waves and fragmented QRS complexes in II, III, and aVF, and inverted T-waves in aVL and V2. The Cornell left ventricular hypertrophy criteria are fulfilled.

\section{Imaging substudy}

Comprehensive cardiac magnetic resonance imaging (CMRI) and 2D strain echocardiography (2DSE) data was available for subset of 65 subjects from the Finnish MYBPC3 imaging study [24], of whom 20 were control subjects and 45 MYBC3-Gln1061X variant carriers without (G $+/ \mathrm{LVH}-n=18)$ or with $\mathrm{LVH}(\mathrm{G}+/ \mathrm{LVH}+n=27)$. The methodology of echocardiography and CMRI for the substudy has been previously published and is summarized in the Methods section of the
Supplementary material [24]. The G+/LVH+ patients were further divided into two groups according to HCM phenotype: mild ( $n=12$ ) and moderate $(n=15)$ HCM. Patients with moderate HCM were defined as having at least one of the following variables $\geq 75$ th percentile of the HCM group: CMRI left ventricular maximal wall thickness (75th percentile limit $\geq 25 \mathrm{~mm}$ ), echocardiographic global longitudinal strain $(\geq-13 \%)$, or CMRI measured degree of late gadolinium enhancement (LGE $\geq 22 \%$ of the LV mass). Mild HCM was defined as not fulfilling any of these criteria. 


\section{Statistical analysis}

Categorical variables are expressed as absolute numbers and percentages, and continuous variables as means and standard deviations or medians followed by inter-quartile ranges. Differences between groups were analyzed using the chi-square test or Fisher's exact test for categorical variables and the Mann-Whitney or Kruskal-Wallis test for continuous variables. Binary logistic regression analysis with sex and age adjustment was used to confirm the validity of the novel ECG parameters $\mathrm{RV} 1<\mathrm{RV} 2>\mathrm{RV} 3$ and septal remodeling. The correlation of the extent of ECG pathology as absolute number of findings to imaging and laboratory parameters in the imaging substudy was analyzed with Spearman rank correlation. A two-tailed p-value of $<0.05$ was considered statistically significant for all tests. All calculations were performed with the SPSS software (IBM SPSS Statistics for Windows, Version 21.0. Armonk, NY, IBM Corp.).

\section{Results}

Baseline

The baseline characteristics and conventional echocardiography findings of the three study groups are presented in Table 1 and Fig. 2A. There were more male subjects in the $\mathrm{G}+/ \mathrm{LVH}+$ group

A Distribution of ECG pathology in all subjects $(n=170)$

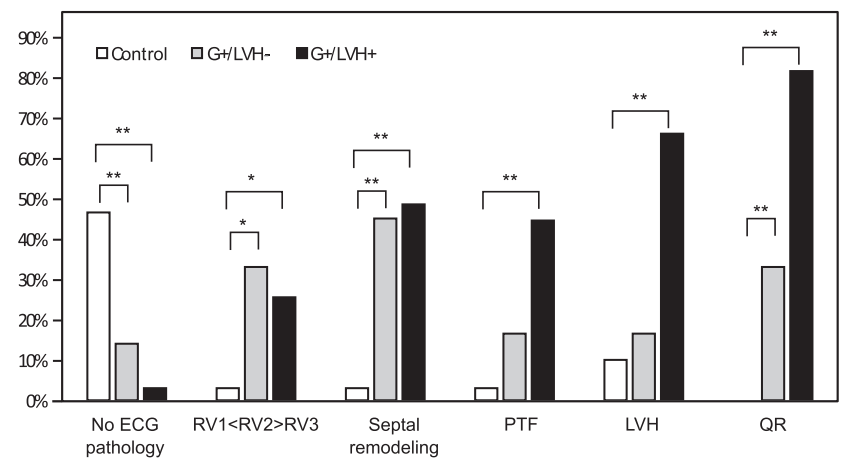

B Distribution of ECG pathology in mutation carriers $(n=140)$

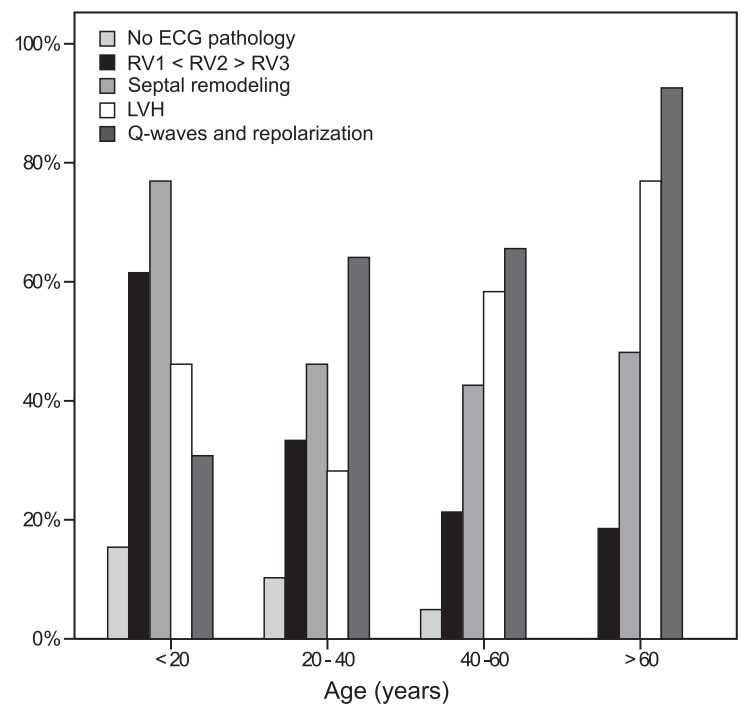

Fig. 2. Distribution of ECG pathology in A) all subjects $(n=170),{ }^{*}=p<0.05$ and ${ }^{* *}=p<$ 0.001 for difference between indicated group and control group; B) in the mutation carriers $(\mathrm{G}+/ \mathrm{LVH}+$ and $\mathrm{LVH}-, n=140)$ stratified by age group. $\mathrm{LVH}=\mathrm{LV}$ hypertrophy by any used criteria: Sokolow-Lyon criteria, Cornell voltage-duration product or Romhilt-Estes point score, $\mathrm{QR}=\mathrm{Q}$ waves and repolarization abnormalities. compared to the control and $\mathrm{G}+/ \mathrm{LVH}$ - group. The subjects in $\mathrm{G}+/$ $\mathrm{LVH}+$ group were also older compared to the $\mathrm{G}+/ \mathrm{LVH}-$ group (48 \pm 14 vs $33 \pm 15$ years, $\mathrm{p}<0.001$ ). The majority of the HCM patients were asymptomatic (78\%). By definition, the $\mathrm{G}+/ \mathrm{LVH}+$ group had significantly greater maximal wall thickness. The groups were balanced regarding left ventricular dimensions and ejection fraction. Left ventricular outflow tract obstruction was present at rest in 5 (5\%) of HCM patients (a gradient of $>30 \mathrm{~mm} \mathrm{Hg}$ ). The majority of $\mathrm{G}+/ \mathrm{LVH}+$ patients had asymmetric septal hypertrophy (90\%). Eight $(8 \%)$ of the HCM patients had MWT in the lateral wall and one patient had posterior and another patient concentric hypertrophy. Two patients had MWT in the apical segments but also exhibited significant thickening of the whole septal wall and did not represent true apical hypertrophy. No differences in echocardiographic measurements were found between the $\mathrm{G}+/ \mathrm{LVH}-$ and control groups.

\section{ECG}

The ECG pathology findings are summarized in Table 2. There were 14 subjects (47\%) in the control group with no ECG pathology in comparison to $6(14 \%)$ and $3(3 \%)$ in the $\mathrm{G}+/ \mathrm{LVH}-$ and $\mathrm{G}+/ \mathrm{LVH}+$ groups, respectively $(\mathrm{p}<0.001)$. Thus the finding of a completely normal ECG was uncommon in all mutation carriers including $\mathrm{G}+/ \mathrm{LVH}-$ subjects. The median number of ECG pathology was 1 (range 0-6) in the $\mathrm{G}+/ \mathrm{LVH}-$ and $5(0-9)$ in the $\mathrm{G}+/ \mathrm{LVH}+$ groups. A normal ECG with no identified pathology had a sensitivity of $47 \%$ and a specificity of $96 \%$ to discern control subjects from HCM mutation carriers. Q waves and repolarization abnormalities $(\mathrm{QR})$ were common findings in the $\mathrm{G}+/ \mathrm{LVH}+$ group. The presence of $\mathrm{Q}$ waves, ST depression and T-wave inversion was marked also in the $\mathrm{G}+/ \mathrm{LVH}-$ group (33\%). ST depressions were associated with inverted T waves in 34/50 (68\%) of the $\mathrm{G}+/ \mathrm{LVH}+$ patients. Conventional ECG parameters of hypertrophy (Sokolow-Lyon and Cornell criteria) identified true LVH quite modestly in the $\mathrm{G}+/ \mathrm{LVH}+$ group. The presence of P-terminal force, was a

Table 2

ECG characteristics of the study groups.

\begin{tabular}{|c|c|c|c|c|}
\hline & \multirow{2}{*}{$\frac{\text { Control }}{(n=30)}$} & \multirow{2}{*}{$\frac{\mathrm{G}+/ \mathrm{LVH}-}{(n=42)}$} & \multirow{2}{*}{$\frac{\mathrm{G}+/ \mathrm{LVH}+}{(n=98)}$} & \multirow[t]{2}{*}{ p-Value } \\
\hline & & & & \\
\hline ECG pathology & $1(0-4)$ & $1(0-6)$ & $5(0-9)^{*}, \dagger$ & $<0.001$ \\
\hline No ECG pathology & $47 \%(14)$ & $14 \%(6)^{*}$ & $3 \%(3)^{*}, \dagger$ & $<0.001$ \\
\hline Major criteria & $3 \%(1)$ & $36 \%(15)^{*}$ & $90 \%(88)^{*}, \dagger$ & $<0.001$ \\
\hline$Q$ waves and repolarization & 0 & $33 \%(14)^{*}$ & $82 \%(80)^{*}, \dagger$ & $<0.001$ \\
\hline Q waves & 0 & $21 \%(9)^{*}$ & $38 \%(37)^{*}$ & $<0.001$ \\
\hline Q V2-V6 & & $7 \%(3)$ & $15 \%(15)$ & 0.031 \\
\hline Q II, III, aVF & & $10 \%(4)$ & $16 \%(16)$ & 0.035 \\
\hline Q I, aVL & & $2 \%(1)$ & $16 \%(16)$ & 0.004 \\
\hline ST depression & 0 & $7 \%(3)$ & $51 \%(50)^{*}, \dagger$ & $<0.001$ \\
\hline T-wave inversion & 0 & $2 \%(1)$ & $44 \%(43)^{*}, \dagger$ & $<0.001$ \\
\hline Prolonged QTc & 0 & $7 \%(3)$ & $18 \%(18)^{*}$ & 0.006 \\
\hline Absent Q V5-6 & $7 \%(2)$ & $12 \%(5)$ & $15 \%(15)$ & 0.539 \\
\hline Giant T-waves & $10 \%(3)$ & $17 \%(7)$ & $6 \%(6)$ & 0.179 \\
\hline \multicolumn{5}{|l|}{ Novel ECG criteria } \\
\hline $\mathrm{RV} 1<\mathrm{RV} 2>\mathrm{RV} 3$ & $3 \%(1)$ & $33 \%(14)^{*}$ & $26 \%(25)^{*}$ & 0.005 \\
\hline Septal remodeling & $3 \%(1)$ & $45 \%(19)^{*}$ & $49 \%(48)^{*}$ & $<0.001$ \\
\hline Maximal R-wave not in V4-6 & $7 \%(2)$ & $19 \%(8)$ & $23 \%(23)$ & 0.122 \\
\hline Hypertrophy & $10 \%(3)$ & $17 \%(7)$ & $66 \%(65)^{*}, \dagger$ & $<0.001$ \\
\hline Sokolow-Lyon criteria & $10 \%(3)$ & $2 \%(1)$ & $15 \%(15)$ & 0.079 \\
\hline Cornell criteria & 0 & $7 \%(3)$ & $40 \%(39)^{*}, \dagger$ & $<0.001$ \\
\hline Romhilt-Estes score $\geq 4$ & $3 \%(1)$ & $14 \%(6)$ & $55 \%(54)^{*}, \dagger$ & $<0.001$ \\
\hline \multicolumn{5}{|l|}{ Other ECG features } \\
\hline P-terminal force & $3 \%(1)$ & $17 \%(7)$ & $45 \%(44)^{*}, \dagger$ & $<0.001$ \\
\hline $\mathrm{QRS}>100 \mathrm{~ms}$ & 0 & $5 \%(2)$ & $22 \%(22)^{*}, \dagger$ & $<0.001$ \\
\hline Fragmented QRS & $27 \%(8)$ & $21 \%(9)$ & $46 \%(45)^{\dagger}$ & 0.011 \\
\hline Prolonged PQ & 0 & $2 \%(1)$ & $11 \%(11)$ & 0.032 \\
\hline Axis deviation & $3 \%(1)$ & $19 \%(8)$ & $1 \%(1)^{*}$ & 0.002 \\
\hline
\end{tabular}

ECG pathology presented as median and range in parentheses. All other variables presented as percentage and number of patients in parentheses.

${ }^{*} \mathrm{p}<0.05$ for difference between indicated group and control group.

$\dagger^{\dagger} \mathrm{p}<0.05$ for difference between $\mathrm{G}+/ \mathrm{LVH}+$ and $\mathrm{G}+/ \mathrm{LVH}-$ groups. 
common finding in HCM patients and also present in $17 \%$ of $\mathrm{G}+/ \mathrm{LVH}-$ subjects. No significant differences between the groups were observed in the following parameters (not shown in the table): RI + SIII $>25 \mathrm{~mm}, \mathrm{RI}+$ SIII-RIII-SI $>17 \mathrm{~mm}, \mathrm{~S}>\mathrm{R}$ V4, PRWP, reverse R-wave progression in leads V1-V3.

The novel ECG criteria of RV1 $<$ RV2 $>$ RV3 (Fig. 1) and septal remodeling were significantly more common in mutation carriers without LVH than in control subjects ( $33 \%$ vs $3 \%, p=0.006$ and $45 \%$ vs $3 \%, p<$ 0.001 , respectively) and in $\mathrm{G}+/ \mathrm{LVH}+$ subjects compared with control subjects ( $26 \%$ vs $3 \%, p=0.015$ and $49 \%$ vs $3 \%, p<0.001$, respectively). In univariate binary logistic regression, with age and sex as covariates, both $\mathrm{RV} 1<\mathrm{RV} 2>\mathrm{RV} 3$ and septal remodeling retained their statistical significance $(\mathrm{OR}=13.3, \mathrm{p}=0.018$ for $\mathrm{RV} 1<\mathrm{RV} 2>\mathrm{RV} 3$ and $\mathrm{OR}=21.8, \mathrm{p}=$ 0.004 for septal remodeling).

Fig. 2B illustrates the different ECG findings stratified by age groups in the mutation carriers with and without LVH $(G+, n=140)$. $\mathrm{RV} 1<\mathrm{RV} 2>\mathrm{RV} 3$ and septal remodeling were more prevalent in younger subjects and, in contrast, the ECG findings of hypertrophy and QR were more pronounced in the older age groups.

\section{Imaging substudy}

The baseline characteristics of the imaging substudy groups are presented in Supplementary Tables 1 and 2. CMRI derived ejection fraction and LV end diastolic volume were normal in all study groups. Typical imaging findings in subjects with mild and moderate HCM were increased septal and maximal wall thickness, increased CMRI LV mass, reduced diastolic function and global longitudinal strain (GLS), and presence of LGE that increased with advanced disease. The prevalence of $\mathrm{RV} 1<\mathrm{RV} 2>\mathrm{RV} 3$, septal remodeling and other key pathologic ECG features according to $\mathrm{HCM}$ phenotype are illustrated in Fig. 3. $\mathrm{RV} 1<\mathrm{RV} 2>\mathrm{RV} 3$ was more prevalent in $\mathrm{G}+/ \mathrm{LVH}-$ and mutation carriers with mild LVH than in patients with moderate or severe HCM. Other ECG findings were more prevalent in the moderate HCM group compared to the mild HCM and $\mathrm{G}+/ \mathrm{LVH}-$ groups. No significant correlations of the novel ECG parameters $\mathrm{RV} 1<\mathrm{RV} 2>\mathrm{RV} 3$ and septal remodeling to imaging findings related to the septum or distribution of wall thickness in segmental strain, LGE or MWT values were found. The correlations of the extent of ECG pathology (0-15) to imaging and laboratory variables in gene mutation carriers with and without LVH are presented in Table 3, showing strong association with E/Em, GLS,

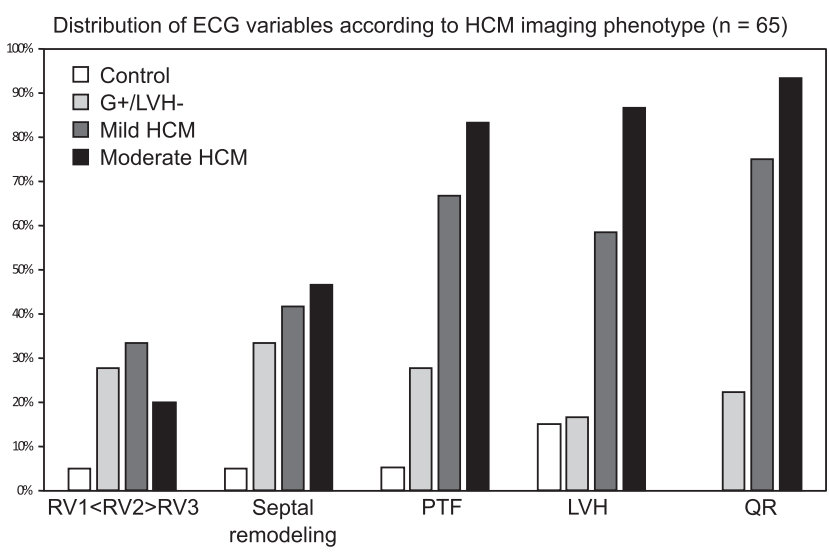

Fig. 3. Distribution of ECG variables according to HCM geno- and phenotype in the imaging substudy subjects $(n=65)$ : Control, G+/LVH-, G+/Mild HCM, G+/Moderate HCM Moderate HCM determined as MWT and/or GLS and/or LGE $\geq 75$ th percentile and mild $\mathrm{HCM}$ as not fulfilling these criteria. RV1 $<\mathrm{RV} 2>\mathrm{RV} 3$ is more prevalent in $\mathrm{LVH}-$ and mild HCM subjects whereas presence of septal remodeling increases with changes related to HCM. LVH = LV hypertrophy by any used criteria: Sokolow-Lyon criteria, Cornel voltage-duration product or Romhilt-Estes point score, PTF $=$ P-terminal force $\geq 0.04 \mathrm{~mm} \cdot \mathrm{s}, \mathrm{QR}=\mathrm{Q}$ waves and repolarization abnormalities.
Table 3

Correlation of variables to the extent of ECG pathology.

\begin{tabular}{lll}
\hline Variable & \multicolumn{2}{l}{ ECG pathology } \\
\cline { 2 - 3 } & $\rho$ & $\mathrm{p}$ \\
\hline ECHO E/E' & 0.58 & $<0.001$ \\
ECHO global systolic longitudinal strain & 0.56 & $<0.001$ \\
ECHO mechanical dispersion & 0.58 & $<0.001$ \\
CMRI LGE extent & 0.62 & $<0.001$ \\
CMRI LV mass index & 0.58 & $<0.001$ \\
CMRI maximal wall thickness & 0.76 & $<0.001$ \\
NT-proBNP & 0.62 & $<0.001$ \\
\hline
\end{tabular}

Spearman rank correlation of echocardiographic (ECHO) and cardiac magnetic resonance imaging (CMRI) variables and NT-proBNP to the extent of ECG pathology in subjects with the HCM-causing variant (0-15). LGE = late gadolinium enhancement.

mechanical dispersion, LGE, LV mass index, MWT, and levels of circulating NT-proBNP.

The ECG finding of QR had good sensitivity (95\%) and relatively good specificity (84\%) for the presence of LGE (95\% of patients with LGE had QR compared to $16 \%$ of patients without LGE, $\mathrm{p}<0.001$ for Fisher's exact test). There were no significant correlations between the localization of LGE and localized ECG-findings such as Q waves or T-wave inversion in the anterior or inferior leads.

\section{The ability of ECG findings to differentiate between study groups}

Table 4 shows the diagnostic value of ECG parameters and their combination for differentiating mutation carriers with and without LVH from the control subjects. The major ECG criteria [22] performed well in differentiating $\mathrm{G}+/ \mathrm{LVH}+$ patients from control subjects, with a sensitivity of $90 \%$ and specificity of $97 \%$. The novel parameters of $\mathrm{RV} 1<\mathrm{RV} 2>\mathrm{RV} 3$ and septal remodeling did not improve the diagnostic sensitivity of the previously proposed criteria in differentiating $\mathrm{G}+$ / $\mathrm{LVH}+$ patients from control subjects. Conventional variables such as the major criteria or QR had modest sensitivity to differentiate between $\mathrm{G}+/ \mathrm{LVH}-$ and control subjects. In contrast, RV1 $<\mathrm{RV} 2>\mathrm{RV} 3$ and septal remodeling both had incremental value in the identification of mutation carriers without LVH. When used in conjunction with QR the sensitivity was $52 \%$ and $64 \%$, respectively, with a specificity of $97 \%$.

\section{Discussion}

The main findings of this study are: 1) a normal ECG is rare (3\%) in HCM patients with TPM1-Asp175Asn or MYBPC3-Gln1061X variants in a comprehensive ECG analysis; 2) a normal ECG is also uncommon (14\%) in G+/LVH - subjects; 3 ) the extent of ECG pathology correlates with severity of the HCM phenotype; 4) novel ECG parameters $\mathrm{RV} 1<\mathrm{RV} 2>\mathrm{RV} 3$ and septal remodeling were very specific to carriers of

Table 4

Effectiveness of ECG variables to separate $\mathrm{G}+/ \mathrm{LVH}+$ and $\mathrm{G}+/ \mathrm{LVH}-$ groups from the control group.

\begin{tabular}{lllll}
\hline & Sensitivity (\%) & Specificity (\%) & PPV (\%) & NPV (\%) \\
\hline G+/LVH+ $(\mathrm{n}=98)$ & & & & \\
Major criteria $(\geq 1)$ & $90 \%$ & $97 \%$ & $99 \%$ & $74 \%$ \\
QR & $82 \%$ & $100 \%$ & $100 \%$ & $63 \%$ \\
RV1 $<$ RV2>RV3 & $26 \%$ & $97 \%$ & $96 \%$ & $28 \%$ \\
Septal remodeling & $49 \%$ & $97 \%$ & $98 \%$ & $37 \%$ \\
G+/LVH- $(\mathrm{n}=42)$ & & & & \\
Major criteria $(\geq 1)$ & $36 \%$ & $97 \%$ & $94 \%$ & $52 \%$ \\
QR & $33 \%$ & $100 \%$ & $100 \%$ & $52 \%$ \\
RV1 $<$ RV2>RV3 & $33 \%$ & $97 \%$ & $93 \%$ & $51 \%$ \\
RV1 $<$ RV2>RV3 and/or QR & $52 \%$ & $97 \%$ & $96 \%$ & $59 \%$ \\
Septal remodeling & $45 \%$ & $97 \%$ & $95 \%$ & $56 \%$ \\
Septal remodeling and/or QR & $64 \%$ & $97 \%$ & $96 \%$ & $66 \%$ \\
\hline
\end{tabular}

$\mathrm{NPV}=$ negative predictive value, $\mathrm{PPV}=$ positive predictive value, $\mathrm{QR}=\mathrm{Q}$ waves and repolarization abnormalities. 
pathogenic variants with and without LVH and 5) these novel parameters with the combination of $\mathrm{Q}$ waves and repolarization abnormalities exhibited good sensitivity and excellent specificity to differentiate the $G$ $+/ \mathrm{LVH}-$ group from the control group.

\section{Study findings in the context of current literature}

The novel ECG criteria RV1 $<\mathrm{RV} 2>\mathrm{RV} 3$ and septal remodeling proposed in this study have good specificity to differentiate between carriers of pathogenic variants for HCM and the control group. Previous reports on the ECG findings of $\mathrm{G}+/ \mathrm{LVH}$ - subjects have been varied and longitudinal follow-up data is scarce $[7,8,10]$. Some smaller scale studies have reported Q waves and repolarization abnormalities [9], while in others there have been very limited ECG findings [7]. The largest published $\mathrm{G}+/ \mathrm{LVH}$ - cohort to date of 76 subjects with a number of different variants, reported Q waves and repolarization abnormalities in $25 \%$ of subjects, a finding that was a specific (98\%) marker for young $\mathrm{LVH}-$ variant carriers ( $42 \%$ of subjects in the study were $<18$ years old ) [8]. In the current study Q waves were present in $21 \%$ of $\mathrm{G}+/ \mathrm{LVH}$ - subjects compared with none in the control group.

Previously the low sensitivity and mostly limited specificity of ECG variables to differentiate $\mathrm{G}+/ \mathrm{LVH}-$ subjects from healthy control individuals has made their use in clinical practice challenging. In this study the combination of $\mathrm{RV} 1<\mathrm{RV} 2>\mathrm{RV} 3$ or septal remodeling with $\mathrm{Q}$ waves and repolarization abnormalities resulted in a significant improvement of the sensitivity of the ECG analysis to over $50 \%$ with a concurrent specificity of $97 \%$. Application of the RV $1<\mathrm{RV} 2>\mathrm{RV} 3+\mathrm{QR}$ criteria especially is relatively easy in clinical daily practice and could be used in the screening of HCM families.

According to our study, ECG criteria for HCM identified pathogenic variant carriers with LVH with high sensitivity, specificity and positive likelihood ratio and compared well to a previous study with a similar age distribution [10]. The extent of ECG pathology and degree of hypertrophy and dysfunction of the left ventricle correlated with the extent of ECG pathology, which corroborates a previous finding [25]. We also found that GLS, a sensitive marker of LV dysfunction, and NT-proBNP, an established marker of disease severity and prognosis in HCM, both correlate strongly to the extent of ECG pathology. These findings are of importance in the clinical follow-up of HCM patients, as the ECG is an inexpensive and reproducible test.

In our study, the Sokolow-Lyon criteria performed suboptimally in identifying $\mathrm{LVH}$, with only $15 \%$ fulfilling the criteria in the $\mathrm{G}+/ \mathrm{LVH}+$ group and $10 \%$ in the control group. The Cornell criteria were positive in $40 \%$ and the Romhilt-Estes score in $55 \%$ of the hypertrophic patients - results that were in line with previous studies [26]. None of the LVH criteria differed significantly in prevalence between the $\mathrm{G}+/ \mathrm{LVH}-$ and control groups.

\section{Possible pathophysiological mechanisms}

The novel ECG parameter proposed in this study, the RV1 $<$ RV2 $>$ RV3 sign, represents a disorderly distribution of the maximal R-wave amplitudes in leads V1-V3. Normally the R-wave amplitude increases from V1 to V3. Although not specifically reported, previous publications have shown subjects with the RV1 $<$ RV2 $>$ RV3 pattern - both HCM patients with LVH in the age range of 10 to 69 years $[27,28]$ and in pathogenic variant carriers without echocardiographic ventricular hypertrophy $[4,6]$.

The RV1 $<$ RV2 $>$ RV3 finding is more prevalent in young subjects (Fig. 2B) and in those with mild HCM (Fig. 3). It is possible that this reflects an early stage of the developing disease as a segmental structural change of the interventricular septum and is later substituted with other more pronounced ECG changes due to significant hypertrophy and fibrosis. However, even with comprehensive modern imaging echocardiographic and CMRI methods, we could not identify significant correlation between the RV1 $<$ RV2 $>$ RV3 sign and characteristics of the interventricular septum of $\mathrm{G}+/ \mathrm{LVH}-$ subjects.

In our previous report, the presence of septal remodeling identified DCM patients either with the lamin $\mathrm{A} / \mathrm{C}$ variant ( $82 \%$ prevalence) or no identified gene substrate (21\%) compared to control subjects $(0 \%)$ [23]. Thus it is not a specific finding for HCM and instead might reflect general LV remodeling of patients with cardiomyopathies of different etiologies. No clear correlation of the location of LGE to Q waves was found in this study reflecting the results of previous larger scale CMRI reports $[12,25]$. On the other hand, the presence of $Q$ waves and repolarization abnormalities was significantly more prevalent in patients with LGE.

\section{Limitations of the study}

We studied carriers of two different pathogenic variants of HCM: MYBPC3-Gln1061X and TPM1-Asp175Asn. Both are prevalent in the Finnish HCM population and as such are probably similar to other pathogenic MYBPC3 and TPM1 variants causing HCM. Therefore, we would presume that the results are generalizable to the spectrum of carriers of pathogenic variants in these two genes. The results of this study have to interpret with caution when discussing the diagnostics and screening of families without a known pathogenic variant. The imaging subset sample size was limited and included only subjects with the MYBPC-Gln1061X variant. Thus, the results of the imaging study have to be interpreted with caution.

\section{Conclusions}

Our study shows that $\mathrm{G}+/ \mathrm{LVH}+$ patients can be identified with high sensitivity, specificity and predictive values. Conventional ECG was also valuable in the identification of $\mathrm{G}+/ \mathrm{LVH}-$ subjects from control subjects using the novel ECG parameters of RV1 $<$ RV2 $>$ RV3 and septal remodeling in combination with $\mathrm{Q}$ waves and repolarization abnormalities. This finding needs to be further evaluated in larger patient cohorts, but holds promise as a clinically feasible tool to identify subjects with preclinical changes of HCM and aid in the screening and follow-up of relatives of HCM patients.

\section{Acknowledgements}

The authors want to express their gratitude for Sini Weckström, RN, and Satu Nenonen, RN, for assistance in data collection.

\section{Funding}

The study was supported by grants from the Aarno Koskelo Foundation, the Finnish Cultural Foundation, Special Governmental Subsidy, the Academy of Finland, the Finnish Cardiovascular Research Foundation, Kuopio University Hospital, and the Finnish Medical Foundation.

\section{Disclosure of interest}

None.

\section{Appendix A. Supplementary data}

Supplementary data to this article can be found online at https://doi. org/10.1016/j.jelectrocard.2018.07.009.

\section{References}

[1] Maron BJ, Haas TS, Murphy CJ, Ahluwalia A, Rutten-Ramos S. Incidence and causes of sudden death in U.S. college athletes. J Am Coll Cardiol 2014;63:1636-43. https:// doi.org/10.1016/j.jacc.2014.01.041 
[2] Sabater-Molina M, Pérez-Sánchez I, Hernández Del Rincón JP, Gimeno JR. Genetics of hypertrophic cardiomyopathy: a review of current state. Clin Genet 2017;93:3-14. https://doi.org/10.1111/cge.13027.

[3] Elliott PM, Anastasakis A, Borger MA, Borggrefe M, Cecchi F, Charron P, et al. 2014 ESC guidelines on diagnosis and management of hypertrophic cardiomyopathy: the task force for the diagnosis and management of hypertrophic cardiomyopathy of the European Society of Cardiology (ESC). Eur Heart J 2014;35:2733-79. https:// doi.org/10.1093/eurheartj/ehu284.

[4] Shimizu M, Ino H, Yamaguchi M, Terai H, Hayashi K, Kiyama M, et al. Chronologic electrocardiographic changes in patients with hypertrophic cardiomyopathy associated with cardiac troponin 1 mutation. Am Heart J 2002;143:289-93.

[5] Charron P, Dubourg O, Desnos M, Isnard R, Hagege A, Millaire A, et al. Diagnostic value of electrocardiography and echocardiography for familial hypertrophic cardiomyopathy in a genotyped adult population. Circulation 1997;96:214-9.

[6] Mogensen J, Murphy RT, Kubo T, Bahl A, Moon JC, Klausen IC, et al. Frequency and clinical expression of cardiac troponin I mutations in 748 consecutive families with hypertrophic cardiomyopathy. J Am Coll Cardiol 2004;44:2315-25. https:// doi.org/10.1016/j.jacc.2004.05.088.

[7] Havndrup O, Bundgaard H, Andersen PS, Allan Larsen L, Vuust J, Kjeldsen K, et al. Outcome of clinical versus genetic family screening in hypertrophic cardiomyopathy with focus on cardiac beta-myosin gene mutations. Cardiovasc Res 2003;57:347-57.

[8] Lakdawala NK, Thune JJ, Maron BJ, Cirino AL, Havndrup O, Bundgaard H, et al. Electrocardiographic features of sarcomere mutation carriers with and without clinically overt hypertrophic cardiomyopathy. Am J Cardiol 2011;108:1606-13. https://doi. org/10.1016/j.amjcard.2011.07.019.

[9] Konno T, Shimizu M, Ino H, Yamaguchi M, Terai H, Uchiyama K, et al. Diagnostic value of abnormal Q waves for identification of preclinical carriers of hypertrophic cardiomyopathy based on a molecular genetic diagnosis. Eur Heart J 2004;25: 246-51. https://doi.org/10.1016/j.ehj.2003.10.031.

[10] Konno T, Shimizu M, Ino H, Fujino N, Hayashi K, Uchiyama K, et al. Phenotypic differences between electrocardiographic and echocardiographic determination of hypertrophic cardiomyopathy in genetically affected subjects. J Intern Med 2005;258: 216-24. https://doi.org/10.1111/j.1365-2796.2005.01539.x.

[11] Jääskeläinen P, Heliö T, Aalto-Setälä K, Kaartinen M, Ilveskoski E, Hämäläinen L, et al. Two founder mutations in the alpha-tropomyosin and the cardiac myosin-binding protein $\mathrm{C}$ genes are common causes of hypertrophic cardiomyopathy in the Finnish population. Ann Med 2013;45:85-90. https://doi.org/10.3109/07853890.2012. 671534.

[12] Dumont CA, Monserrat L, Soler R, Rodríguez E, Fernandez X, Peteiro J, et al. Interpretation of electrocardiographic abnormalities in hypertrophic cardiomyopathy with cardiac magnetic resonance. Eur Heart J 2006;27:1725-31. https://doi.org/10. 1093/eurheartj/ehl101.

[13] Xiao HB, Gibson DG. Absent septal q wave on electrocardiogram: a forgotten marker of myocardial disease. Int J Cardiol 1996;53:1-4.

[14] Surawicz B, Knilans T. Chou's electrocardiography in clinical practice: adult and pediatric; 2008
[15] Molloy TJ, Okin PM, Devereux RB, Kligfield P. Electrocardiographic detection of left ventricular hypertrophy by the simple QRS voltage-duration product. J Am Coll Cardiol 1992;20:1180-6.

[16] Gubner R. Electrocardiographic criteria of left ventricular hypertrophy. Arch Intern Med (Chic) 1943;72:196-209. https://doi.org/10.1001/archinte.1943. 00210080052005.

[17] Lewis T. Observations upon ventricular hypertrophy with special reference to one or other chamber. Heart 1914:5:367-402.

[18] Ostman-Smith I, Wisten A, Nylander E, Bratt E-L, Granelli AD-W, Oulhaj A, et al. Electrocardiographic amplitudes: a new risk factor for sudden death in hypertrophic cardiomyopathy. Eur Heart J 2010;31:439-49. https://doi.org/10.1093/eurheartj/ ehp443.

[19] Romhilt DW, Estes EH. A point-score system for the ECG diagnosis of left ventricular hypertrophy. Am Heart J 1968;75:752-8.

[20] Das MK, Khan B, Jacob S, Kumar A, Mahenthiran J. Significance of a fragmented QRS complex versus a $Q$ wave in patients with coronary artery disease. Circulation 2006; 113:2495-501. https://doi.org/10.1161/CIRCULATIONAHA.105.595892.

[21] Zema MJ, Kligfield P. Electrocardiographic poor R wave progression. I: correlation with the Frank vectorcardiogram. J Electrocardiol 1979;12:3-10.

[22] McKenna WJ, Spirito P, Desnos M, Dubourg O, Komajda M. Experience from clinical genetics in hypertrophic cardiomyopathy: proposal for new diagnostic criteria in adult members of affected families. Heart 1997:77:130-2.

[23] Ollila L, Nikus K, Holmström M, Jalanko M, Jurkko R, Kaartinen M, et al. Clinical disease presentation and ECG characteristics of LMNA mutation carriers. Open Heart 2017;4:e000474. https://doi.org/10.1136/openhrt-2016-000474.

[24] Jalanko M, Tarkiainen M, Sipola P, Jääskeläinen P, Lauerma K, Laine M, et al. Left ventricular mechanical dispersion is associated with nonsustained ventricular tachycardia in hypertrophic cardiomyopathy. Ann Med 2016:1-11. https://doi.org/10.1080/ 07853890.2016 .1186826

[25] Delcrè SDL, Di Donna P, Leuzzi S, Miceli S, Bisi M, Scaglione M, et al. Relationship of ECG findings to phenotypic expression in patients with hypertrophic cardiomyopathy: a cardiac magnetic resonance study. Int J Cardiol 2013;167:1038-45. https:// doi.org/10.1016/j.ijcard.2012.03.074.

[26] Montgomery JV, Harris KM, Casey SA, Zenovich AG, Maron BJ. Relation of electrocardiographic patterns to phenotypic expression and clinical outcome in hypertrophic cardiomyopathy. Am J Cardiol 2005;96:270-5. https://doi.org/10.1016/j.amjcard. 2005.03.058.

[27] Pérez-Riera AR, de Lucca AA, Barbosa-Barros R, Yanowitz FG, de Cano SF, Cano MN, et al. Value of electro-vectorcardiogram in hypertrophic cardiomyopathy. Ann Noninvasive Electrocardiol 2013;18:311-26. https://doi.org/10.1111/anec.12067.

[28] Ohmoto-Sekine Y, Suzuki J-I, Shimamoto R, Yamazaki T, Tsuji T, Nagai R, et al. Gender-specific clinical characteristics of deep Q waves in hypertrophic cardiomyopathy. Gend Med 2007;4:274-83. 